\title{
In Response to Sexual Dysfunction in Men with Type II Diabetes Mellitus: Prevalence and Severity_An Observational Study
}

\author{
Sattik Siddhanta \\ Bengal Physician Journal (2021): 10.5005/jp-journals-10070-7064
}

Respected Sir,

At the outset, I must appreciate the original article titled "Sexual Dysfunction in Men with Type II Diabetes Mellitus: Prevalence and Severity-An Observational Study" published in the last edition of your esteemed journal. Diabetes mellitus (DM) is one of the commonest chronic noncommunicable diseases encountered globally. Epidemiological studies have clearly documented that sexual dysfunction (SD) particularly erectile dysfunction (ED) is one of the major complications of DM among males. In fact, ED can serve as a potential coronary artery disease (CAD) risk equivalent. However, in comparison with the other microvascular and macrovascular complications of DM, SD is often neglected and studies evaluating SD in males is very rare not only worldwide but also in eastern India. Hence, such a research article addressing this area of complication of DM is highly praiseworthy and pertinent.

The authors conducted a single-center, hospital-based, cross-sectional study over a period of 12 months and included 109 type II diabetic men who were married and aged between 18 and 65 years irrespective of the duration of the disease. However, as the legal minimum age for marriage is 21 years for men in India, approved by the amendment of the Child Marriage Restraint Act, 1929, in 1978 and recommended by the Special Marriage Act, 1954, and the Prohibition of Child Marriage Act, 2006, it would have been ideal to include males aged above 21 years in the study. ED is detected by having male patients' complete standardized questionnaires investigating their sexual function. Though various pretested and predesigned standardized questionnaires are available to assess ED, one of the most practical questionnaires that are administered is the International Index of Erectile Function (IIEF)-5, which the authors have used as a study tool in their study. It consists of items 5, 15, 4, 2, and 7 from the full-scale IIEF-15; a sum score of 21 or less indicates the presence of ED. Advancing age, presence of overweight or obesity, and addiction like alcohol and smoking are strong accessory determinants of ED. Despite including young and middle-aged subjects in the study (only $4.6 \%$ of diabetic males were aged greater than 60 years), the authors obtained the prevalence of ED $89.9 \%$ comprising $62.4 \%$ severe ED which is a huge number, thereby implying a public health pressing problem. When ED occurs in younger males, it is associated with a greater increase in the risk of future cardiac events as compared to its first
Department of General Medicine, IPGMER and SSKM Hospital, Kolkata, West Bengal, India

Corresponding Author: Sattik Siddhanta, Department of General Medicine, IPGMER and SSKM Hospital, Kolkata, West Bengal, India, Phone: +91 9433151126, e-mail: drcalmed@gmail.com

How to cite this article: Siddhanta S. In Response to Sexual Dysfunction in Men with Type II Diabetes Mellitus: Prevalence and Severity-An Observational Study. Bengal Physician Journal 2021;8(3):79-80.

Source of support: Nil

Conflict of interest: None

detection in older males. Therefore, younger men with early-onset ED may be the ideal candidates for intensive cardiovascular (CV) risk factor screening and medical interventions. History of addiction has not been mentioned in the demographic profile of the study participants. In contrast to other similar studies, another significant point as reflected in the study is that though ED had a positive correlation with the duration of type II DM, yet it showed no relation with the severity of hyperglycemia and glycemic variability. This finding is in contrast to the inferences revealed from other similar studies worldwide. Patients with poor metabolic control are at a greater risk of developing ED than those with good metabolic control. Poor metabolic control is associated with an increased risk of long-term macro- and microvascular complications that might have a greater impact on the occurrence of ED. In other words, the severity of the illness (DM) is presumed to be raised among patients with poor metabolic control that concomitantly increases the risk of diabetic complications, including SD. Presence of other comorbidities like hypertension, dyslipidemia, and cardiovascular disease raises the odds ratio of SD in DM by 5.07. However, no such data among the study participants were available from the study. The study population included patients attending a tertiary care center and may not be representative of those seen by general practitioners or not seeking medical attention at all. Furthermore, the results obtained from specific clinical settings cannot be easily generalized to wider populations. The study reported the burden of ED particularly in the Indian context that would help to expand an individual's sphere of knowledge in the highly neglected and less discussed yet highly pertinent segment. In addition, as the study

(o) The Author(s). 2021 Open Access This article is distributed under the terms of the Creative Commons Attribution 4.0 International License (https://creativecommons. org/licenses/by-nc/4.0/), which permits unrestricted use, distribution, and non-commercial reproduction in any medium, provided you give appropriate credit to the original author(s) and the source, provide a link to the Creative Commons license, and indicate if changes were made. The Creative Commons Public Domain Dedication waiver (http://creativecommons.org/publicdomain/zero/1.0/) applies to the data made available in this article, unless otherwise stated. 
was conducted in a tertiary healthcare center, it is presumed to be helpful to acquire reliable clinical data. Another point which needs to be mentioned in the study is whether all possible strategies have been applied to reduce bias like recruiting male interviewers and underway the interview in the most private room as the study might have introduced social desirability bias due to the sensitivity of the research question and the nature of the data collection technique (face-to-face interviewer-administered questionnaire). As these are the factors that were not mentioned, the study might have been exposed to confounding effects. Further, the study did not measure the fasting serum testosterone level of the participants which could be another limitation of the study to show the effect of diabetes on men's sexuality.

Thanking You. 